\title{
Nucleating and retarding effects of nanohydroxyapatite on the crystallization of poly(butylene terephthalate-co-alkylene dicarboxylate)s with different lengths
}

Nina Heidarzadeh ${ }^{1}$, Mehdi Rafizadeh ${ }^{1}$, Faramarz Afshar Taromi ${ }^{1}$, Jordi Puiggali ${ }^{2}$, Luis J. del

$$
\text { Valle }^{2}
$$

${ }^{1}$ Department of Polymer Engineering and Color Technology, Amirkabir University of Technology, 15875-441, Tehran, IRAN

${ }^{2}$ Departament d'Enginyeria Química, Universitat Politècnica de Catalunya, Escola d'Enginyeria Barcelona Est (EEB), c/ Eduard Maristany 10-14, Barcelona E-08019, SPAIN

\footnotetext{
${ }^{1}$ Author for correspondence: mehdi@aut.ac.ir
} 


\section{ABSTRACT}

New biodegradable and biocompatible composites are continuously developed for biomedical applications (e.g. from drug delivery devices to tissue engineering scaffolds). Properties of such systems may depend on their morphology and structure, which are attained after their processing and therefore, the study of the crystallization kinetics has a particular relevance. The crystallization kinetics of hydroxyapatite-filled poly(butylene terephthalate-co-alkylene dicarboxylate)s has been studied under non-isothermal conditions, using a wide range of cooling rates and different kinetic models. Based on our results, nanohydroxyapatite (nHAp) particles were found to effectively act as additional nucleation sites for poly(butylene terephthalate-co-succinate) (PBST), giving rise to an increased crystallization rate with respect to pure PBST. However, the overall growth rate of HAp nanocomposites decreased compared to the corresponding homopolymers with longer aliphatic dicarboxylic acids (i.e. adipic and sebacic acid derivatives). In order to clarify this point, the activation energy for non-isothermal crystallization was evaluated using the Friedman method and significant differences were observed, suggesting a disturbing effect of nanoparticles on the motion of molecular chains that hindered their capability to reach the growing crystallization front. Isoconversional methods provided a good understanding of the kinetics of the crystallization process and significant information regarding the activation energy, relative crystallinity, and global and local Avrami exponents.

Keywords: Non-isothermal crystallization, isokinetic and isoconversional method, poly(alkylene dicarboxylate), biodegradable polymer, hydroxyapatite. 


\section{INTRODUCTION}

In recent years, biodegradable and biocompatible polymer composites reinforced with bioactive ceramics have attracted a great deal of interest, due to their high potential for various applications such as drug delivery devices, nerve regeneration guides, orthopedic implants for tissue reconstruction, bone fixation and tissue engineering scaffolds [1-6]. For instance, composites of bioactive ceramics (e.g., calcium phosphate, hydroxyapatite (HAp) and bioglass) and polymers have been extensively used as potential materials for bone-tissue replacement [7-9]. Specifically, hydroxyapatite $\left(\mathrm{Ca}_{10}\left(\mathrm{PO}_{4}\right)_{6}(\mathrm{OH})_{2}\right)$ is mainly employed in orthopedic and dental reconstruction, because of its excellent biocompatibility and osteointegration properties [10]. Bioactive nanohydroxyapatite (nHAp) can be used as a filler for the development of nanocomposites based on biodegradable polymer matrices. These biodegradable nanocomposites have great potential for applications such as orthopedic implants, intersomatic cages for spinal arthrodesis and osteosynthetic plates in fracture healing [11].

The most widely used biodegradable polymer matrices include polylactide, polyhydroxyalkanoates, polyanhydrides, polyorthoesters and their copolymers. Their preparation, bioactivity, biodegradability, mechanical properties, modification and utilization of their composites with nHAp have been the focus of many studies. It has been indicated for example that the incorporation of HAp or tricalcium phosphate bioceramics into biodegradable polyhydroxyalkanoates reduces their crystallinity, increases the degradation rate of the biopolymer and facilitates the reconstruction of new tissues [12]. Aggregation of nanoparticles at high filler contents can cause local stress concentration, internal cracks and weak mechanical properties [13], and therefore the amounts of incorporated nanoparticles have been limited to values around 2-5 wt-\% [14]. Different solutions have been proposed to reduce such aggregation. For example, the 
use of amphiphilic molecules has been reported to favor the dispersion of highly problematic carbon nanotubes [15]. However, improvement of mechanical properties has not been always achieved and even a plasticization effect of the added molecules on the polymeric matrix has been observed.

Despite the numerous studies concerning biodegradable nanocomposites, there is still a noncompletely resolved crucial point relative to their crystallization kinetics, since a complex influence of nanoparticles has been reported. Understanding the mechanism and kinetics of the crystallization of these materials is vital for determining their ultimate applicability and for the design of new polymer composite structures with desirable properties for biomedical applications. The knowledge of the parameters affecting crystallization is crucial for optimization of the processing conditions and properties of the end product [16], especially for fast crystallizing biodegradable polymers $[17,18]$ and polyesters in particular.

Preparation of new copolyalkylene dicarboxylates offers a key opportunity for increasing the range of degradable materials and even generating a set of products with easily tunable properties. Thus, different types of poly(butylene succinate) (PBS) copolymers incorporating terephthalic acid units have been recently developed to achieve a good compromise between the degradability caused by the presence of aliphatic ester segments and the mechanical properties provided by the aromatic ester moieties. Furthermore, the use of copolymers has clear advantages among which, the following benefits can be highlighted: enhancement of biodegradability due to the increase in the amorphous content, cost reduction, increase in the commercial offer and even the capability of modifying final properties. Incorporation of comonomers has also a great influence on the melting behavior, sample crystallinity, lamellar surface morphology, and consequently the enzymatic degradability [19-22]. 
Several studies have been recently focused on the crystallization process of random aliphaticaromatic copolyesters [23-32]. In general, isothermal analyses have been performed since they provide accurate data considering the well-defined crystallization conditions. However, since crystallization during processing is always non-isothermal, a scientific and technological challenge is introduced to the kinetic study in such a continuously changing environment [33-35] that can be modeled as a sequence of infinitesimally small isothermal steps.

The main focus of the present work was to study the effect of HAp nanoparticles on the nonisothermal crystallization kinetics of biodegradable copolyesters derived from 1,4-butanediol, terephthalic acid and aliphatic dicarboxylate acids differing in the length of their polymethylene segment (i.e. succinic, adipic and sebacic acids for 2, 4 and 8 methylene groups, respectively). To this end, the molar ratio between aromatic and aliphatic dicarboxylates (i.e. 65 mol- $\%$ ) as well as the nHAp content (i.e. 2 wt-\%) were kept constant. In the rest of this paper, the unloaded and HAp loaded samples will be referred to as PBXT and PBXT-nHAp, respectively, where X indicates the alkylene dicarboxylate unit (i.e. S, Ad and Se for succinate, adipate and sebacate, respectively).

\section{EXPERIMENTAL}

\section{Materials}

The polyesters studied in this work were synthesized from terephthalic acid (TA) (Shahid Tondgooyan Petrochemical Complex, Mahshahr, Iran), and succinic acid (SA), adipic acid (AdA), sebacic acid (SeA) and 1,4-butanediol (BDO) that were supplied by Daejung Chemical \& Metal Co., Ltd, Korea. Titanium tetrabutoxide (TBT) as the polycondensation catalyst was purchased from Merck Co., Darmstadt, Germany. All other materials and solvents were of analytical grade. Nanohydroxiapatite (n-HAp) (with a diameter of $20 \mathrm{~nm}$ ) was purchased from Beijing DK nano technology Co., Ltd, China. 


\section{Synthesis of copolyesters and preparation of nanocomposites}

The aliphatic copolyesters were prepared by a two-stage melt polycondensation process in a stainless steel batch reactor [36]. In brief, a mixture of $65 \mathrm{~mol} \% \mathrm{TA}$ and the appropriate aliphatic dicarboxylic acid (i.e. SA, AdA or SeA) were introduced to the reactor, together with an excess of 1,4-butanediol (i.e. the acid:diol molar ratio was $1.17: 1$ ). The temperature was then set to $180^{\circ} \mathrm{C}$, $190{ }^{\circ} \mathrm{C}$ and $200-215{ }^{\circ} \mathrm{C}$ for SA, AdA and SeA derivatives, respectively, under a nitrogen atmosphere. The reaction conversion was followed by weighting the evolved and condensed water at regular $(15 \mathrm{~min})$ time intervals. The reaction was stopped when no more water was recovered (approximately after $180 \mathrm{~min}$ ).

In the second polycondensation step, TBT catalyst was added (1.4 mmol for $1 \mathrm{~mol}$ of dicarboxylic acid). The reaction mixture was initially kept for 10 min at $200{ }^{\circ} \mathrm{C}$ under a nitrogen atmosphere and then vacuum (20 mbar) was slowly applied over a time period of about 20 min to minimize oligomer sublimation, which is a potential problem during melt polycondensation. The temperature was finally increased to $250{ }^{\circ} \mathrm{C}-255^{\circ} \mathrm{C}$ for an additional time period of 2.5 hours.

Copolymers were purified through precipitation with 1,1,1,3,3,3-hexafluoroisopropanol (HFIP) solution, being subsequently washed with water, methanol and ether, several times. Nanocomposites were prepared through solvent casting of previously sonicated copolymer solutions in HFIP containing $2.5 \mathrm{wt}-\%$ of dispersed nHAp.

\section{Gel permeation chromatography (GPC)}

Molecular mass were estimated via gel permeation chromatography (GPC) using a liquid chromatograph (Shimadzu, model LC-8A, Tokyo, Japan) equipped with an Empower computer program (Waters, Milford, MA USA). A PL HFIP gel column (Polymer Lab, Böblingen, Germany) and a refractive index detector (Shimadzu RID-10A, Tokyo, Japan) were employed. 
The polymer was dissolved and eluted in HFIP containing CF3COONa (0.05 M) at a flow rate of $1 \mathrm{~mL} \mathrm{~min}^{-1}$ (injected volume $100 \mu \mathrm{L}$, sample concentration $2.0 \mathrm{mg} \mathrm{mL}^{-1}$ ). The number and mass average molecular mass were calculated using poly methyl methacrylate standards.

\section{Nuclear magnetic resonance (NMR)}

${ }^{1} \mathrm{H}$ NMR spectra were obtained via a Bruker AMX-500 spectrometer operating at a frequency of $500 \mathrm{MHz}$ for protons while deuterated chloroform was used as the solvent.

\section{Differential scanning calorimetry (DSC)}

DSC analysis was performed using a Mettler Toledo DSC1 device, equipped with a refrigerated cooling system (RCS). The instrument was calibrated with indium and zinc standards. For each test, about 9-10 mg of the sample was placed in a sealed aluminum pan and heated at $20{ }^{\circ} \mathrm{C} / \mathrm{min}$. To remove the thermal history, the sample was held in the melt state for $3 \mathrm{~min}$ and subsequently non-isothermally crystallized by cooling to room temperature at a rate of $10{ }^{\circ} \mathrm{C} / \mathrm{min}$. A second

heating run (i.e. $5,10,15$ and $20{ }^{\circ} \mathrm{C} \min ^{-1}$ ) was finally performed to characterize the melting behavior of such crystallized samples.

\section{Thermogravimetric analysis (TG)}

Thermal degradation was studied at a heating rate of $10{ }^{\circ} \mathrm{C} \mathrm{min}{ }^{-1}$ with around $5 \mathrm{mg}$ samples in a Q50 thermogravimetric analyzer (TG) of TA Instruments, under a flow of dry nitrogen. Test temperatures were in the range of 50 to $600{ }^{\circ} \mathrm{C}$. 


\section{RESULTS AND DISCUSSION}

\section{Synthesis and comonomer composition}

Copolymers with practically the same molecular mass were prepared to evaluate the effect of molecular structure on the crystallization kinetics of the derived HAp nanocomposites. Thus, the influence of molecular mass on crystallization rates could be ignored. The number average molecular mass of purified copolyesters varied between 9,200 and 9,700 $\mathrm{g} \mathrm{mol}^{-1}$, and the polydispersity index remained in the typical range of polycondensation samples (i.e., between 2.4 and 2.5) (as shown in Table 1). Obviously, these values remained unchanged when nHAp was incorporated through solvent casting.

${ }^{1} \mathrm{H}-\mathrm{NMR}$ analysis indicated that the three copolymers had similar aromatic contents and random comonomer distributions. Figure 1a shows the NMR spectrum of PBSeT as a representative sample.

Signal areas at 8.14-8.17 ppm (terephthalate units, T) (proton a) and 2.42-2.79 ppm (aliphatic dicarboxylate units, A) (proton d) were used to determine the corresponding mole fractions ( $f_{T}$ and $f_{a}$ ). Specifically, in all cases $f_{a}$ values were close to 0.35 in full agreement with the feed ratio of aromatic and aliphatic dicarboxylate monomers. The proton resonances of methylenes adjacent to the dicarboxylic acid units (i.e. signals $\mathrm{b}$ and $\mathrm{c}$ at $4.0-4.5 \mathrm{ppm}$ and $1.80-2.35 \mathrm{ppm}$, respectively) were split into multiplets, due to the four adjacent possible sequences for the butylene units: the homo units (TBT and $\mathrm{ABA}$ ) and the hetero ones (ABT and $\mathrm{TBA})$. Integration of these signals allowed calculating the degree of randomness, $r$, as reported in previous papers [37]. Values (as shown in Table 1) were always close to 1 , which is a characteristic of random copolymers. 


\section{Thermal stability of nanocomposites}

Thermogravimetric analysis results confirmed the successful incorporation of n-HAp, as the nanocomposite char yield determined at $550-600{ }^{\circ} \mathrm{C}$ increased by around $2 \%$ with respect to the value determined for the neat copolymer (Figure $1 \mathrm{~b}$ for the sebacic acid derivative). Such value is consistent with the loaded amount of n-HAp. TG curves showed also a destabilizing effect of HAp nanoparticles, since they have a small fraction of hydroxyl groups that can promote the thermal degradation of polyesters. It is notable that the onset of degradation of the representative sebacic acid derivative decreased from $310^{\circ} \mathrm{C}$ to $250^{\circ} \mathrm{C}$ and even that degradation occurred according to a complex process where different steps could be envisaged. In any case, nanocomposites were sufficiently stable to be processed from the melt state with no evidence of degradation (i.e. the onset of degradation was more than $95^{\circ} \mathrm{C}$ higher than the melting temperature).

\section{Melting of PBXT and PBXT-nHAP samples}

Random copolymers constituted by two crystallizable repeat units can display different melting and crystallization behaviors depending on the miscibility of the comonomers and the ability to share crystal lattices [38]. Comonomer units can be totally excluded from the crystalline regions into the amorphous phase or alternatively, a cocrystallization can be observed.

In order to investigate the melting behavior of the synthesized copolyesters, DSC heating scans were performed on melt crystallized samples as shown in Figure 2. In all cases, only the peaks associated with the PBT aromatic crystalline phase were detected. This feature could be well justified considering the highly predominant terephthalate content and also the fact that the aromatic phase should crystallize first in the crystallization process from the melt state. The differences between the melting temperatures of the corresponding aromatic and aliphatic 
homopolymers were highly significant (i.e., $223^{\circ} \mathrm{C}, 115^{\circ} \mathrm{C}, 58^{\circ} \mathrm{C}$ and $63^{\circ} \mathrm{C}$ for PBT, PBS, PBAd and PBSe, respectively [39-42]).

In all cases, melting temperatures of the synthesized copolymers were in the range of $145-166^{\circ} \mathrm{C}$, being significantly lower than the value previously reported for the PBT homopolymer $\left(223{ }^{\circ} \mathrm{C}\right)$ [39]. Therefore, a similar amount of aliphatic dicarboxylate units should be incorporated in the aromatic phase of the studied copolymers. Two endothermic peaks that appeared overlapped could be clearly detected for the succinic and adipic derivatives and were even envisaged for the sebacic derivative (see arrows in blue in Figure 2). This feature could be interpreted as a typical lamellar reorganization process where the thin lamellae melts and recrystallizes, giving rise to thicker crystals with a higher melting point. In any case, differences between melting temperatures and enthalpies were minimal, considering the presence of different dicarboxylic acids and even HAp nanocrystals. The PBSeT-nHAp sample exhibited the greatest variation (with a $6{ }^{\circ} \mathrm{C}$ decrease) compared to the neat PBSeT sample. In addition, the sebacate derivative showed the highest change in the melting enthalpy, which was decreased by almost $5 \mathrm{~J} \mathrm{~g}^{-1}$ after nanoparticle addition. After crystallization of the aromatic crystalline phase during cooling, the aliphatic phase could develop once its crystallization temperature was reached. It was reasonable that this second crystallization process became hindered by the previously formed crystals and consequently the corresponding low temperature melting peaks were not detected.

The amorphous phase segregation of the aliphatic component should be more significant for the crystallization of PBAdT and PBSeT copolymers due to their extremely low melting temperatures (i.e. $58-63^{\circ} \mathrm{C}$ with respect to the $115^{\circ} \mathrm{C}$ characteristic value of PBS). The exclusion of adipate and sebacate should render the formation of amorphous pockets at the crystal growth front, and hence affect the crystallization behavior. 


\section{Non-isothermal crystallization behavior}

In Figure 3, the non-isothermal melt-crystallization exotherms of PBXT copolymers and their corresponding composites containing nHAp have been shown for four different crystallization rates $(\Phi)$. Based on these curves, the peak temperature $\left(T_{\mathrm{c}}\right)$ at which the sample exhibited the fastest crystallization, and the onset temperature $\left(T_{0}\right)$ [43] could be easily obtained. Both $T_{\mathrm{c}}$ and $T_{0}$ increased as expected, when $\Phi$ decreased. Logically, when samples were cooled at a low rate, they had enough time to form the necessary nuclei and crystallize. However, when a higher $\Phi$ value was used, the motion of copolyester chains could not follow the cooling process and therefore, a higher undercooling was required to initiate the crystallization.

For a given $\Phi$ value, all $T_{\mathrm{c}}$ values for PBST-nHAp were consistently higher than those determined for the pure PBST, suggesting that the addition of nHAp promotes the crystallization of the copolyester. On the contrary, the incorporation of nHAp in adipate and sebacate derivatives caused a decrease in both $T_{\mathrm{c}}$ and $T_{0}$ with respect to the values found for the neat homopolymers. This effect was much more pronounced for the sebacic acid derivative.

The changes in the crystallization temperatures could be related to the combination of two opposite factors. On one hand, nHAp particles serve as additional nucleating agents [44] and consequently, their introduction promotes the crystallization as observed for PBST. On the other hand, the addition of a filler may restrict the motion of molecular chains and even affect the secondary nucleation constant. The crystallization process is in this case hindered, as was observed for PBAdT and PBSeT nanocomposites. The increase in the aliphatic segment length should enhance the chain flexibility and decrease its polar character. The experimental evidence was that nHAp particles seemed to display a retarding effect for aliphatic/aromatic copolyesters with more apolar aliphatic comonomers, in other words it became more difficult to incorporate molecular segments 
to the crystal surfaces of the predominant PBT phase. This point may be evidenced by the significant observed increase in the secondary nucleation constant, as will be subsequently demonstrated for the sebacate derivative. Retarding effects caused by the incorporation of different nanofillers have been reported in the literature, being explained by the reduced chain diffusion of the polymer due to new interactions between polymer chains and the filler surfaces $[45,46]$. Logically, this effect not only should be enhanced by increasing the filler content but also should depend on the polymer physicochemical characteristics as can be deduced from the results obtained from a series of polymers such as those investigated in the present work.

\section{Non-isothermal crystallization kinetics}

The relative crystallinity, $\alpha_{\mathrm{t}}$, as a function of crystallization temperature can be calculated from the DSC cooling runs, as the following:

$$
\alpha_{t}=\frac{\int_{T_{0}}^{T}\left(\frac{\mathrm{d} H_{c}}{\mathrm{~d} T}\right) \mathrm{d} T}{\int_{T_{0}}^{T_{\infty}}\left(\frac{\mathrm{d} H_{c}}{\mathrm{~d} T}\right) \mathrm{d} T}
$$

where $T_{0}$ and $T_{\infty}$ denote the onset and end of crystallization temperatures, respectively, and $\mathrm{d} H \mathrm{c} /$ $\mathrm{d} T$ is the heat flow rate. The crystallization temperature, $T$, can be converted to crystallization time, $t$, using the cooling rate, $\phi$, and the following equation:

$$
t=\frac{T_{0}-T}{\Phi}
$$

The relationship between $\alpha_{t}$ and $t$ for PBST, PBAdT and PBSeT and the corresponding nanocomposites can be obtained via combining equations 1 and 2 . Figure 4 compares the conversion curves for all studied copolymers. Logically, when a higher cooling rate was employed a shorter time was required for crystallization completion. The half-time of crystallization $\left(t_{1 / 2}\right)$ 
could be easily obtained from Figure 4 at $\alpha_{t}=0.5$ and became a single parameter for comparing the overall rate of crystallization. The values summarized in Table 3 obviously indicate that $t_{1 / 2}$ decreases with the cooling rate increase, but no clear trend could be found for the effect of nHAp incorporation.

The crystallization rate parameter (CRP) [44] is useful to compare the relative crystallization rates of different polymer systems and get more conclusive results regarding the effect of n-HAp. CRP is determined from the slopes of the $t_{1 / 2}{ }^{-1}-\Phi$ plot (Figure 5). The higher values indicate a faster crystallization.

CRP values for PBST, PBAdT and PBSeT were 0.026, 0.035 and $0.049 \mathrm{~K}^{-1}$ whereas $0.037,0.029$ and $0.025 \mathrm{~K}^{-1}$ values determined for PBST-nHAp, PBAdT-nHAp and PBSeT-nHAp, respectively. Results were logically consistent with the former discussion based on the simple $T_{0}$ and $T_{\mathrm{c}}$ parameters and again indicated a nucleating (for PBST) or retarding (for PBSeT and in a minor degree for PBAdT) effect of nHAp.

\section{Non-isothermal crystallization analyses}

Isokinetic and isoconversional analytical methods have been previously applied to describe the non-isothermal crystallization kinetics of the selected polymers and their composites [47-49]. The former method is based on the isokinetic hypothesis, in which the kinetic parameters are constant during the whole crystallization process. Specifically, the modified Avrami, Jeziorny, Mo and Nakanura analyses can be considered. The isoconversional methods assume that the crystallization rate at a constant extent of conversion is only a function of temperature. Therefore the kinetic parameters derived from isoconversional methods depend on the degree of conversion at different temperatures and times $[47,48]$. 


\section{Modified Avrami and Jeziorny analyses}

The Avrami model describes a time dependent relative crystallinity function, $\alpha_{t}$, for a nonisothermal crystallization process that can be written as the following $[50,51]$ :

$$
\alpha_{t}=1-\exp \left(-Z_{t} t^{n}\right)
$$

where $\mathrm{Z}_{\mathrm{t}}$ denotes the growth rate constant; $n$ is the global Avrami exponent which depends on the nucleation mechanism and shape of the grown crystallites, $t$ is the time duration of crystallization process and $\alpha_{t}$ is the relative crystallinity.

A normalized rate constant, $k=\mathrm{Z}_{\mathrm{t}}^{1 / n}$, is also usually evaluated for comparison purposes since its dimension $\left(\right.$ time $\left.^{-1}\right)$ is independent of the Avrami exponent.

Avrami exponent for isothermal experiments has a physical sense, as it is uniquely related to the nucleation rate and the crystallite growth morphology. The interpretation of non-isothermal crystallization is not very clear, because it reflects the occurrence of processes that could be different (e.g. depending on the primary nucleation and crystallization regimens) or even be greatly influenced by the secondary crystallization [48].

In order to avoid the dependency of $Z_{t}$ on the cooling rate, a new constant $Z_{\mathrm{c}}$ has been proposed by Jeziorny [52].

$$
\ln Z_{c}=\frac{\ln Z_{t}}{\Phi}
$$

where $\Phi$ is the cooling rate and $Z_{c}$ is a modified crystallization rate constant.

Table 3 summarizes the values of $n, Z_{t}, Z_{c}$ and $k$ for all copolyesters and their nanocomposites. It could be observed that almost all $n$ values were in the range of $4.3-3.1$ and increased with increasing $\Phi$. The change in $n$ could indicate the differences in crystal growth geometry and the type of nucleation, since it is well established for isothermal crystallizations that an $n$ value of 3 indicates the spherulitic growth from instantaneous nuclei, whereas a value of 4 represents the 
spherulitic growth from sporadic nuclei [53]. In this way, nucleation seems to become time dependent as crystallization becomes faster or alternatively the contribution of a constrained crystallization should become more significant at lower rates. Results summarized in Table 3 also indicate that $n$ values for different neat copolyesters and their related nanocomposites were similar for a given cooling rate. It is notable that morphological changes should not be expected due to the small variation in the crystallization temperature and consequently, the alterations in the Avrami exponent should be related to the type of nucleation [54]. In this way, the presence of HAp nanoparticles did not change the type (i.e. instantaneous or sporadic) of nucleation of the neat copolyesters, although they obviously increased the primary nucleation.

Parameters $Z_{\mathrm{t}}, Z_{\mathrm{c}}$ and $k$ strongly depended on $\Phi$ and logically increased when increasing the rate. The evolution of $k$ values was also similar to those observed for the experimental $t_{1 / 2}{ }^{-1}$ values. This agreement between direct experimental values (i.e. $t_{1 / 2}{ }^{-1}$ ) and theoretical ones (i.e. $k$ ) could be considered as an indication of a well performed Avrami analysis.

\section{Mo analysis}

Non-isothermal crystallization has also been studied by applying the well-known Ozawa equation that directly considers the effect of cooling rate and correlates the cooling rate with temperature, time, and morphology [55]:

$$
\alpha_{t}=1-\exp \left(\frac{-K(T)}{\Phi^{m}}\right)
$$

where $K(T)$ and $m$ represent a function of the process temperature and the Ozawa exponent, respectively. $K(T)$ is referred to as the crystallization rate and indicates how fast the crystallization occurs. $m$ has a physical meaning similar to the Avrami exponent and therefore depends on the crystal dimension. 
However, Ozawa analysis is limited because of deviations from the theoretical model due to the fact that crystallinities at a given temperature can correspond to the primary or secondary processes depending on the cooling rate.

Mo and coworkers [55] have successfully combined Ozawa and Avrami equations and obtained a new equation to describe the non-isothermal crystallization kinetics of several polymers [56, 57]:

$$
\ln \Phi=\ln F(T)-a \ln t
$$

where the parameter $F(T)=(K(T) / \mathrm{Z} t)^{1 / m}$ refers to the value of the cooling rate, which has to be chosen at a unit crystallization time when the system under study reaches a certain degree of crystallinity; $a=(n / m)$ is the ratio between the Avrami $(n)$ and Ozawa $(m)$ exponents. According to Eq. 6, at a given degree of crystallinity, the plot of $\ln \Phi$ versus $\ln t$ will be a straight line, and the value of $(T)$ and $a$ can be obtained by the intercept and the slope of the line, respectively. Mo parameters for the studied copolyesters and nanocomposites have been summarized in Table 4. For all samples, straight lines were obtained (data not shown), indicating that the non-isothermal crystallization was well described by the Mo model.

As shown in Table 4, the $F(T)$ values increased with the relative degree of crystallinity, while $a$ showed only a slight variation. $F(T)$ is considered as a parameter that indicates the polymer crystallization rate. Lower $F(T)$ values are associated to higher crystallization rates under nonisothermal crystallization conditions $[16,43]$. Based on the results, $F(T)$ increased with increasing $\alpha_{t}$, which suggests that a higher cooling rate is needed to obtain a higher $\alpha_{t}$ value in unit crystallization time. Moreover, $F(T)$ values for PBST-nHAp were lower than those determined for the neat PBST, being indicative of the faster crystallization kinetics. This observation was in full agreement with the previous observations that implied the nucleation effect of nHAp in such system. On the contrary, $F(T)$ values for PBAdT-nHAp and especially for PBSeT-nHAp were 
higher than those deduced for the corresponding neat copolyesters as expected for the retarding effect of nHAp.

$a$ values were almost constant for a given sample, but slightly decreased (from 1.33 to 0.80 ) or increased (from 0.95 to 1.79 ) for the neat copolyesters or the nanocomposites, respectively, when the length of the aliphatic dicarboxylate increased. In this regard, $a$ values of nanocomposites were lower and higher than those of the corresponding neat copolyester for the succinate and sebacate derivatives, respectively.

\section{Nakamura analysis}

The Nakamura nonlinear regression was also applied to gain insight into the non-isothermal crystallization behavior [58].

The quiescent non-isothermal crystallization rate constant, $k(T)$, could be determined by fitting the differential form of the Nakamura's model $[58,59]$ to the experimental $\mathrm{d} \alpha / \mathrm{d} t$ values that could be obtained from a plot of the degree of crystallinity, $\alpha$, versus $T$ and the value of the Avrami exponent, $n$ :

$$
\frac{\mathrm{d} \alpha}{\mathrm{d} t}=n k(T)(1-\alpha)[-\ln (1-\alpha)]^{\frac{n-1}{n}}
$$

On the other hand $k(T)$ could be described by the Hoffman-Lauritzen equation [60]:

$$
k(T)=(\ln 2)^{1 / n}\left(\frac{1}{t_{1 / 2}}\right)_{0} \exp \left(\frac{-U / R}{T-T_{\infty}}\right) \exp \left(\frac{-K_{g}}{T \Delta T f}\right)
$$

where $\left(1 / t_{1 / 2}\right)_{0}$ is a pre-exponential factor that includes all temperature independent terms; $U$ is the activation energy for the transport of crystallizing units across the phase boundary; $K_{g}$ is the secondary nucleation constant; $T_{\infty}$ is the temperature below which molecular transport ceases; $R$

is the universal gas constant; $\Delta T=T_{m}{ }^{0}-T$ is the degree of supercooling, $f=2 T /\left(T^{0}{ }_{\mathrm{m}}+T\right)$ is a 
correction factor accounting for the reduction in the latent heat of fusion as the temperature is decreased, and $T_{m}{ }^{0}$ is the equilibrium melting temperature which is $165^{\circ} \mathrm{C}$ for PBST and PBSTnHAp, $173^{\circ} \mathrm{C}$ for PBAdT and PBAdT-nHAp and $169^{\circ} \mathrm{C}$ for PBSeT and PBSeT-nHAp. The nonlinear regression method was used to obtain the parameters $\left(1 / t_{1 / 2}\right)_{0}$ and $K_{\mathrm{g}}$ in equation 8 . In this way, $k(T)$ given by Eq. 8 was introduced into Eq. 7 and the differential form of Nakamura's model was fitted to the experimental $\mathrm{d} \alpha / \mathrm{dt}$ versus temperature data.

Kinetic features at low supercoolings are basically governed by the nucleation term, and consequently the crystallization rates are relatively insensitive to the $U^{*}$ and $T_{\infty}$ parameters. Therefore, typical values of $6276 \mathrm{~J} \mathrm{~mol}^{-1}$ and $T_{\infty}=T_{g}-30 \mathrm{~K}$ were assumed in calculations [61]. A $T_{\infty}$ of -57 and $-37{ }^{\circ} \mathrm{C}$ was employed for all calculations assuming a $T_{g}$ of -27 and $-7{ }^{\circ} \mathrm{C}$ for the three copolymers with the same aromatic content and the corresponding nanocomposites [36].

Table 5 summarizes the calculated Hoffman and Lauritzen parameters along with the correlation coefficient when an Avrami exponent equal to a typical value of 3 was assumed. The calculated data indicated that PBAdT-nHAp and PBSeT-n HAp presented higher $K_{\mathrm{g}}$ values compared to pure PBAdT and PBSeT. Therefore, these nanocomposites had higher barriers for the secondary nucleation in agreement with the previously suggested retarding effect and the observed crystallization rates. On the contrary, for pure PBST the energy barrier for the crystal growth was higher than that determined for the nanocomposite. Crystallization occurred over a limited temperature range, making this impossible to distinguish between the crystallization regimes or explain the differences in the nucleation constant based on a crystallization regime change.

\section{Determination of activation energy from isoconversional methods}

Local activation energy, $\Delta E_{\alpha}$, which represents the activation energy for a crystallized volume fraction of $\alpha$, was also calculated by using the isoconversional method to describe the kinetic 
process. Specifically, the differential isoconversional method of Friedman [62], the integral Kissinger-Akahira-Sunose (KAS) [63] or the advanced integral isoconversional method of Vyazovkin [64] have been considered since nowadays appear as the most appropriate approaches for the analysis of non-isothermal melt crystallization events.

The Friedman expression is given by Eq. 10:

$$
\ln \left(\frac{\mathrm{d} \alpha}{\mathrm{d} t}\right)_{\alpha}=C-\frac{\Delta E_{a,(\alpha)}}{R T_{\alpha}}
$$

where $\mathrm{C}$ is a constant.

According to this method, the $\alpha(t)$ function is obtained from the integration of the experimentally measured crystallization rates, and subsequent differentiating with respect to time to obtain the instantaneous crystallization rate, $\mathrm{d} \alpha / \mathrm{dt}$. The values of $\mathrm{d} \alpha / \mathrm{d} t$ at a specific degree of crystallinity $(\alpha)$ are correlated to the corresponding crystallization temperature at this $\alpha$ value (i.e., $T_{\alpha}$ ). According to Friedman, different effective activation energies are calculated for every degree of crystallinity using the above equation from the slope of the regression line being equal to $\Delta E_{d} / R$. A straight line must be obtained by plotting the left-hand side of Eq. (10) with respect to $1 / T_{\alpha}$, with the slope being equal to $\Delta E_{a} / R$.

Figure 6 shows that the calculated values of $\Delta E_{\mathrm{a}}$ had a small variation over almost the entire range of $\alpha$, except for the values close to the lower and higher extremes. All three nanocomposite samples showed practically constant energies whereas the neat PBAdT and PBSeT samples clearly exhibited energy decreases at high degrees of crystallinity, which mean a faster crystallization process. At a given relative crystallinity, the $\Delta E_{\text {a }}$ values of PBAdT-nHAp and PBSeT-nHAp samples were higher (i.e. less negative) than those observed for pure copolyesters, a finding that was consistent with those deduced from our previous crystallization kinetic analyses. Similarly, 
the values deduced for PBST-nHAp were slightly lower than that calculated for the corresponding neat copolymer.

The simplified Kissinger-Akahira-Sunose (KAS) method has been also proposed to evaluate $\Delta E_{\mathrm{a}}$. In this approximation the kinetic equation can be written as [63]:

$$
\ln \left(\frac{\Phi}{T_{\alpha}^{2}}\right)=C-\frac{\Delta E_{a,(\alpha)}}{R T_{\alpha}}
$$

In Figure 6, the deduced values of $\Delta E_{\mathrm{a}}$ for PBS and PBST-nHAp as representative samples, have been compared. It is worth noting that the results were highly comparable to those obtained from the Friedman analysis and that, again the energy was slightly lower for the nanocomposite in agreement with its faster crystallization.

According to Vyazovkin et al. [65] the most attractive feature of applying isoconversional methods to DSC data is that the resulting $\Delta E_{\mathrm{a}}$ dependencies can be utilized for estimating the parameters of the Lauritzen-Hoffman theory. The extensive experimental measurements carried out by Toda et al. [66] demonstrate that the logarithmic derivative of the microscopic growth rate, $G$, is equivalent to the logarithmic derivative of the heat flow, $\Phi$, according to the following expression:

$$
\frac{\mathrm{d} \ln (G)}{\mathrm{d} T^{-1}}=\frac{\mathrm{d} \ln (\Phi)}{\mathrm{d} T^{-1}}
$$

Vyazovkin and Sbirrazzuoli have used Eq. 12 in association with the well-known LauritzenHoffman equation [60]:

$$
G=G_{0} \exp \left[-\frac{U^{*}}{R\left(T-T_{\infty}\right)}\right] \exp \left[-\frac{K_{g}}{\left.T\left[T_{m}^{0}-T\right)\right]}\right]
$$

to derive the temperature dependency of the effective activation energy of the growth rate. 


$$
\Delta E_{\alpha}=-R \frac{\mathrm{d} \ln (G)}{\mathrm{d}(1 / T)}=U^{*} \frac{T^{2}}{\left(T-T_{\infty}\right)^{2}}+K_{g} R \frac{\left(T_{m}^{0}\right)^{2}-T^{2}-T_{m}^{0} T}{\left(T_{m}^{0}-T\right)^{2} T}
$$

$U^{*}, T_{\infty}$ and $K_{g}$ have been described in previous sections. Logically, typical values of $6276 \mathrm{~J} / \mathrm{mol}$ and $T_{\mathrm{g}}-30 \mathrm{~K}$ can be assumed as previously mentioned.

The effective activation energies at a given conversion can be correlated with temperature as required by equation 14 to get the estimated secondary nucleation constant for different samples. Figure 7 shows the corresponding plots of the activation energy versus temperature for the three copolyesters and their nanocomposites. It is clear that the observed dependency cannot be fitted using a single value of $K_{g}$ since Eq. 14 should render a decreasing activation energy with temperature increase (see Figure 8 for the representative PBST sample). Therefore, the crystallization process should be described by different crystallization regimens as previously suggested for PBT where regimens III and II have been described [67]. This is the main point that could be concluded from the isoconversional analyses and specifically, the nucleation constant values could be estimated as summarized in Table 6 . It is notable that experimental data were obtained over a very limited temperature range and consequently, severe approximations were applied for the rough simulation process (e.g. the ratio between nucleation constants of regimes III and II was always kept close to 2). Table 6 summarizes the estimated nucleation constants for the different studied samples. Based on the results, the nucleation constants for the nanocomposite of the succinate derivative were lower than that determined for the neat copolyester, while an opposite behavior was observed for both adipate and sebacate derivatives. Furthermore, simulation could be performed using a value of the secondary nucleation (regimen II) close to those determined via the Nakamura methodology.

\section{CONCLUSIONS}


In the present study, crystallization under non-isothermal conditions was investigated for different copolymers derived from 1,4-butanediol and mixtures of aromatic and aliphatic dicarboxylic acids. Based on a typical Avrami analysis, PBSeT and PBST showed the greatest and the lowest overall crystallization rates, respectively. All copolyesters showed a change in the crystallization rate after nHAp incorporation, due to its nucleating or retarding effect for the succinic derivative and the other two derivatives, respectively.

Both modified Avrami method and the combination of the Avrami and Ozawa equations proposed by Mo provided a satisfactory description of the experimental data. The values of $t_{1 / 2}$ and $k$ showed that for both neat copolyesters and nanocomposites the crystallization rate increased with increasing $\Phi$.

The isoconversional approach allowed estimating the temperature dependency of the effective activation energy for the non-isothermal hot-crystallization. This approximation was useful to determine the existence of two crystallization regimes. In any case, nHAp particles displayed a retarding effect for aliphatic/aromatic copolyesters with more apolar aliphatic comonomers, a behavior that reflects a greater difficulty of molecular segments to be incorporated into the crystal surfaces of the predominant PBT phase. This point is evidenced by a significant increase of the secondary nucleation constant.

Acknowledgements. J.P. and L.V. are thankful for the supports from MINECO and FEDER (MAT2015-69547-R) and the Generalitat de Catalunya (2014SGR188). 


\section{REFERENCES}

1. Letic-Gavrilovic A, Piattelli A, Abe K. Nerve growth factor $\beta$ (NGF $\beta$ ) delivery via a collagen/hydroxyapatite (Col/HAp) composite and its effects on new bone ingrowth. J Mater Sci Mater Med. 2003;14:95-102.

2. Wang Y-W, Wu Q, Chen J, Chen G-Q. Evaluation of three-dimensional scaffolds made of blends of hydroxyapatite and poly (3-hydroxybutyrate-co-3-hydroxyhexanoate) for bone reconstruction. Biomaterials. 2005;26:899-904.

3. Sotome S, Uemura T, Kikuchi M, Chen J, Itoh S, Tanaka J, et al. Synthesis and in vivo evaluation of a novel hydroxyapatite/collagen-alginate as a bone filler and a drug delivery carrier of bone morphogenetic protein. Mater Sci Eng C. 2004;24:341-7.

4. Mohanna PN, Young RC, Wiberg M, Terenghi G. A composite poly-hydroxybutyrate-glial growth factor conduit for long nerve gap repairs. J Anat. 2003;203:553-65.

5. Chadda H, P.S. S, Satapathy BK, Ray AR. Filler-immobilization assisted designing of hydroxyapatite and silica/ hydroxyapatite filled acrylate based dental restorative composites: Comparative evaluation of quasi-static and dynamic mechanical properties. J Polym Res. 2016;23:197.

6. Pielichowska K. The influence of molecular weight on the properties of polyacetal/hydroxyapatite nanocomposites. Part 1. Microstructural analysis and phase transition studies. J Polym Res. 2012;19:9775.

7. Suebwongnat S, Jianprasert A, Siriphannon P, Monvisade P. Calcium silicate/poly(ethylene terephthalate) biomaterials via ring-opening polymerization. J Polym Res. 2012;19:9985.

8. Chiu D-J, Li Y, Feng C-K, Yang M-R, Chen K-S, Swieszkowski W. Preparation and enhanced mechanical properties of hydroxyapatite hybrid hydrogels via novel photocatalytic polymerization. J Polym Res. 2017;24:227.

9. Wang J. Polyether Ether Ketone. Handb Eng Spec Thermoplast [Internet]. Wiley-Blackwell; 2011 [cited 2018 Oct 19]. p. 55-95. Available from: https://onlinelibrary.wiley.com/doi/abs/10.1002/9781118104729.ch3

10. Jayabalan M, Shalumon KT, Mitha MK, Ganesan K, Epple M. Effect of hydroxyapatite on the biodegradation and biomechanical stability of polyester nanocomposites for orthopaedic applications. Acta Biomater. 2010;6:763-775.

11. Christenson EM, Anseth KS, van den Beucken JJ, Chan CK, Ercan B, Jansen JA, et al. Nanobiomaterial applications in orthopedics. J Orthop Res. 2007;25:11-22.

12. Chen LJ, Wang M. Production and evaluation of biodegradable composites based on PHB-PHV copolymer. Biomaterials. 2002;23:2631-2639.

13. Wang M. Developing bioactive composite materials for tissue replacement. Biomaterials. 2003;24:2133-2151.

14. Bonfield W. Hydroxyapatite-Reinforced Polyethylene as an Analogous Material for Bone Replacementa. Ann N Y Acad Sci. 1988;523:173-177. 
15. Barrau S, Demont P, Perez E, Peigney A, Laurent C, Lacabanne C. Effect of palmitic acid on the electrical conductivity of carbon nanotubes- epoxy resin composites. Macromolecules. 2003;36:96789680.

16. Papageorgiou GZ, Achilias DS, Bikiaris DN. Crystallization kinetics and melting behaviour of the novel biodegradable polyesters poly (propylene azelate) and poly (propylene sebacate). Macromol Chem Phys. 2009;210:90-107.

17. Di Lorenzo ML, Silvestre C. Non-isothermal crystallization of polymers. Prog Polym Sci. 1999;24:917950.

18. Bosq N, Aht-Ong D. Isothermal and non-isothermal crystallization kinetics of poly(butylene succinate) with nanoprecipitated calcium carbonate as nucleating agent. J Therm Anal Calorim. 2018;132:233-49.

19. Díaz A, Katsarava R, Puiggalí J. Synthesis, properties and applications of biodegradable polymers derived from diols and dicarboxylic acids: From polyesters to poly (ester amide) s. Int J Mol Sci. 2014;15:7064-7123.

20. Gan Z, Abe H, Doi Y. Biodegradable poly (ethylene succinate)(PES). 2. Crystal morphology of meltcrystallized ultrathin film and its change after enzymatic degradation. Biomacromolecules. 2000;1:713720.

21. Gan Z, Abe H, Doi Y. Biodegradable poly (ethylene succinate)(PES). 1. Crystal growth kinetics and morphology. Biomacromolecules. 2000;1:704-712.

22. Gan Z, Abe H, Doi Y. Crystallization, melting, and enzymatic degradation of biodegradable poly (butylene succinate-co-14 mol ethylene succinate) copolyester. Biomacromolecules. 2001;2:313-321.

23. Park SS, Chae SH, Im SS. Transesterification and crystallization behavior of poly (butylene succinate)/poly (butylene terephthalate) block copolymers. J Polym Sci Part Polym Chem. 1998;36:147156.

24. Li F, Xu X, Li Q, Li Y, Zhang H, Yu J, et al. Thermal degradation and their kinetics of biodegradable poly (butylene succinate-co-butylene terephthate) s under nitrogen and air atmospheres. Polym Degrad Stab. 2006;91:1685-1693.

25. Wittmann JC, Lotz B. Epitaxial crystallization of aliphatic polyesters on trioxane and various aromatic hydrocarbons. J Polym Sci Polym Phys Ed. 1981;19:1853-1864.

26. Gan Z, Kuwabara K, Yamamoto M, Abe H, Doi Y. Solid-state structures and thermal properties of aliphatic-aromatic poly (butylene adipate-co-butylene terephthalate) copolyesters. Polym Degrad Stab. 2004;83:289-300.

27. Chen Y, Tan L, Chen L, Yang Y, Wang X. Study on biodegradable aromatic/aliphatic copolyesters. Braz J Chem Eng. 2008;25:321-335.

28. Ki HC, Park OO. Synthesis, characterization and biodegradability of the biodegradable aliphaticaromatic random copolyesters. Polymer. 2001;42:1849-1861.

29. Tserki V, Matzinos P, Pavlidou E, Vachliotis D, Panayiotou C. Biodegradable aliphatic polyesters. Part I. Properties and biodegradation of poly (butylene succinate-co-butylene adipate). Polym Degrad Stab. 2006;91:367-376. 
30. Harris JE, Goh SH, Paul DR, Barlow JW. Miscible binary blends containing the polyhydroxy ether of bisphenol-a and various aliphatic polyesters. J Appl Polym Sci. 1982;27:839-855.

31. Papageorgiou GZ, Tsanaktsis V, Papageorgiou DG, Exarhopoulos S, Papageorgiou M, Bikiaris DN. Evaluation of polyesters from renewable resources as alternatives to the current fossil-based polymers. Phase transitions of poly (butylene 2, 5-furan-dicarboxylate). Polymer. 2014;55:3846-3858.

32. Codou A, Guigo N, van Berkel J, De Jong E, Sbirrazzuoli N. Non-isothermal Crystallization Kinetics of Biobased Poly (ethylene 2, 5-furandicarboxylate) Synthesized via the Direct Esterification Process. Macromol Chem Phys. 2014;215:2065-2074.

33. Hu X, Lesser AJ. Non-Isothermal Crystallization of Poly (trimethylene terephthalate)(PTT)/Clay Nanocomposites. Macromol Chem Phys. 2004;205:574-580.

34. Martínez-Palau M, Franco L, Puiggalí J. Isothermal crystallization of poly (glycolic acid-alt-6hydroxyhexanoic acid) studied by DSC and real time synchrotron SAXS/WAXD. Polymer. 2007;48:60186028.

35. Long Y, Shanks RA, Stachurski ZH. Kinetics of polymer crystallisation. Prog Polym Sci. 1995;20:651701.

36. Heidarzadeh N, Rafizadeh M, Taromi FA, del Valle LJ, Franco L, Puiggalí J. Effect of Hydroxyapatite Nanoparticles on the Degradability of Random Poly (butylene terephthalate-co-aliphatic dicarboxylate) s Having a High Content of Terephthalic Units. Polymers. 2016;8:253.

37. Herrera R, Franco L, Rodríguez-Galán A, Puiggalí J. Characterization and degradation behavior of poly (butylene adipate-co-terephthalate) s. J Polym Sci Part Polym Chem. 2002;40:4141-4157.

38. Heidarzadeh N, Rafizadeh M, Taromi FA, del Valle LJ, Franco L, Puiggalí J. Thermal degradation of random copolyesters based on 1, 4-butanediol, terepthalic acid and different aliphatic dicarboxylic acids. Thermochim Acta. 2017;654:101-111.

39. Ou C-F, Chao M-S, Huang S-L. The crystallization behaviors of poly (butylene terephthalate) blended with co [poly (butylene terephthalate-p-oxybenzoate)] copolyesters. Eur Polym J. 2000;36:2665-2670.

40. Nagata M, Kiyotsukuri T, Ibuki H, Tsutsumi N, Sakai W. Synthesis and enzymatic degradation of regular network aliphatic polyesters. React Funct Polym. 1996;30:165-171.

41. Zhao P, Liu W, Wu Q, Ren J. Preparation, mechanical, and thermal properties of biodegradable polyesters/poly (lactic acid) blends. J Nanomater. 2010;2010:4.

42. Glass Transition [Internet]. [cited 2018 Oct 19]. Available from: http://polymerdatabase.com/polymer\%20physics/GlassTransition.html

43. Tang CY, Chen DZ, Tsui CP, Uskokovic PS, Yu PH, Leung MC. Nonisothermal melt-crystallization kinetics of hydroxyapatite-filled poly (3-hydroxybutyrate) composites. J Appl Polym Sci. 2006;102:53885395.

44. Zhang X, Li Y, Lv G, Zuo Y, Mu Y. Thermal and crystallization studies of nano-hydroxyapatite reinforced polyamide 66 biocomposites. Polym Degrad Stab. 2006;91:1202-1207.

45. Li Y, Han C, Bian J, Zhang X, Han L, Dong L. Crystallization and morphology studies of biodegradable poly ( $\varepsilon$-caprolactone)/silica nanocomposites. Polym Compos. 2013;34:131-140. 
46. Chatterjee T, Yurekli K, Hadjiev VG, Krishnamoorti R. Single-walled carbon nanotube dispersions in poly (ethylene oxide). Adv Funct Mater. 2005;15:1832-1838.

47. Bianchi O, Dal Castel C, de Oliveira RV, Bertuoli PT, Hillig E. Nonisothermal degradation of wood using thermogravimetric measurements. Polímeros. 2010;20:395-400.

48. Bianchi O, Martins JDN, Fiorio R, Oliveira RVB, Canto LB. Changes in activation energy and kinetic mechanism during EVA crosslinking. Polym Test. 2011;30:616-624.

49. Guigo N, Van Berkel J, De Jong E, Sbirrazzuoli N. Modelling the non-isothermal crystallization of polymers: Application to poly (ethylene 2, 5-furandicarboxylate). Thermochim Acta. 2017;650:66-75.

50. Avrami M. Kinetics of phase change. I General theory. J Chem Phys. 1939;7:1103-1112.

51. Avrami M. Kinetics of phase change. II transformation-time relations for random distribution of nuclei. J Chem Phys. 1940;8:212-224.

52. Jeziorny A. Parameters characterizing the kinetics of the non-isothermal crystallization of poly (ethylene terephthalate) determined by DSC. Polymer. 1978;19:1142-1144.

53. Christian JW. The theory of transformations in metals and alloys. Newnes; 2002.

54. Chen C, Fei B, Peng S, Zhuang Y, Dong L, Feng Z. Nonisothermal crystallization and melting behavior of poly (3-hydroxybutyrate) and maleated poly (3-hydroxybutyrate). Eur Polym J. 2002;38:1663-1670.

55. Liu T, Mo Z, Wang S, Zhang H. Nonisothermal melt and cold crystallization kinetics of poly (aryl ether ether ketone ketone). Polym Eng Sci. 1997;37:568-575.

56. Xiong H, Gao Y, Li HM. Non-isothermal crystallization kinetics of syndiotactic polystyrenepolystyrene functionalized SWNTs nanocomposites. Lett. 2007;1:416-426.

57. Silvestre C, Pezzuto M, Duraccio D, Mitchell GR, Cimmino S. Quiescent and shear-induced nonisothermal crystallization of isotactic polypropylene-based nanocomposites. Polym Bull. 2017;74:145-165.

58. Patel RM, Spruiell JE. Crystallization kinetics during polymer processing-analysis of available approaches for process modeling. Polym Eng Sci. 1991;31:730-738.

59. Nakamura K, Katayama K, Amano T. Some aspects of nonisothermal crystallization of polymers. II. Consideration of the isokinetic condition. J Appl Polym Sci. 1973;17:1031-1041.

60. Hoffman JD, Davis GT, Lauritzen JI. The rate of crystallization of linear polymers with chain folding. Treatise Solid State Chem. Springer; 1976. p. 497-614.

61. Suzuki T, Kovacs AJ. Temperature dependence of spherulitic growth rate of isotactic polystyrene. A critical comparison with the kinetic theory of surface nucleation. Polym J. 1970;1:82.

62. Friedman HL. Kinetics of thermal degradation of char-forming plastics from thermogravimetry. Application to a phenolic plastic. J Polym Sci Part C Polym Symp. Wiley Online Library; 1964. p. 183195.

63. Akahira T, Sunose T. Method of determining activation deterioration constant of electrical insulating materials. Res Rep Chiba Inst Technol Sci Technol. 1971;16:22-31. 
64. Vyazovkin S, Sbirrazzuoli N. Isoconversional approach to evaluating the Hoffman-Lauritzen parameters $\left(\mathrm{U}^{*}\right.$ and $\mathrm{Kg}$ ) from the overall rates of nonisothermal crystallization. Macromol Rapid Commun. 2004;25:733-738.

65. Vyazovkin S. Some basics en route to isoconversional methodology. Isoconversional Kinet Therm Stimul Process. Springer; 2015. p. 1-25.

66. Toda A, Oda T, Hikosaka M, Saruyama Y. A new method of analysing transformation kinetics with temperature modulated differential scanning calorimetry: application to polymer crystal growth. Polymer. 1997;38:231-233.

67. Supaphol P, Dangseeyun N, Srimoaon P. Non-isothermal melt crystallization kinetics for poly (trimethylene terephthalate)/poly (butylene terephthalate) blends. Polym Test. 2004;23:175-185. 


\section{FIGURE CAPTIONS}

Figure 1. a) ${ }^{1} \mathrm{H}$ NMR spectra of PBSeT with peak assignment. Insets show the multiplets corresponding to the splitting of the $\mathrm{CH}_{2} \mathrm{O}$ and $\mathrm{CH}_{2} \mathrm{CH}_{2} \mathrm{O}$ protons according to the copolymer sequence. b) Thermogravimetric curves of PBSeT (solid lines) and PBSeT-nHAp (dashed lines).

Figure 2. DSC heating scans of pure copolyesters (solid line) and nanocomposites (dashed line) after crystallization from the melt state (i.e. samples were kept at $10{ }^{\circ} \mathrm{C}$ above the fusion temperature for $3 \mathrm{~min}$ to erase thermal history and then cooled at $10{ }^{\circ} \mathrm{C} \mathrm{min}^{-1}$ rate to room temperature).

Figure 3. Non-isothermal crystallization exotherms of the pure copolyesters (solid lines) and the corresponding nanocomposites (dashed lines): a) PBST and PBST-nHAp, b) PBAdT and PBAdTnHAp and c) PBSeT and PBSeT-nHAp. Cooling rates were 5, 10, 15 and $20^{\circ} \mathrm{C} \mathrm{min}^{-1}$ for the traces labelled as (1), (2), (3) and (4), respectively.

Figure 4. Relative crystallinity versus time plots for (a) PBST and PBST-nHAp (b) PBAdT and PBAdT-nHAp and (c) PBSeT and PBSeT-nHAp. Cooling rates were 5, 10, 15 and $20{ }^{\circ} \mathrm{C}$ min $^{-1}$ for the traces labelled as (1), (2), (3) and (4), respectively. Pure copolyesters (red lines) and the corresponding nanocomposites (black lines).

Figure 5. Plots of the reciprocal half-time of crystallization versus the cooling rate for the different neat copolyesters and their nanocomposites with n-HAp.

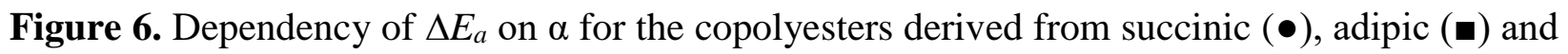
sebacic acids (A) (a) and their corresponding nanocomposites (b) as determined using the Friedman method. As complementary data, the results for succinate derivatives (o) obtained from the KAS method have been also plotted. 
Figure 7. Dependency of $\Delta E_{a}$ on temperature for different copolyesters $(\bullet)$ and their nanocomposites (०). Solid lines represent the values deduced from equation 14.

Figure 8. Experimental dependency of $\Delta E_{a}$ on crystallization temperature for the PBST copolyester $(\bullet)$ and its nanocomposite $(\circ)$. solid and dashed line correspond to the data obtained by equation 14 and the optimized parameters for regime II, dotted and dashdotted line for regime III for PBST and PBST-nHAp respectively. 
Table 1. GPC and spectroscopic data of PBXT samples

\begin{tabular}{cccccc}
\hline Sample & $\boldsymbol{M}_{\mathbf{n}} / \mathbf{g ~ \mathbf { ~ m o l } ^ { \mathbf { 1 } }}$ & $\boldsymbol{M}_{\mathbf{w}} / \mathbf{g ~ m o l}^{\mathbf{- 1}}$ & PDI & $\boldsymbol{f}_{\boldsymbol{a}}^{\mathbf{a}}$ & $\boldsymbol{r}$ \\
\hline PBST & 9500 & 23,100 & 2.43 & 0.35 & 1.03 \\
PBAdT & 9700 & 24,200 & 2.49 & 0.36 & 1.00 \\
PBSeT & 9200 & 22,000 & 2.39 & 0.35 & 1.04 \\
\hline
\end{tabular}

${ }^{\text {a }}$ Determined from ${ }^{1} \mathrm{H}$ NMR spectra as $A_{2.42-2.79} /\left(A_{2.42-2.79}+A_{8.14-8.17}\right)$ where $A$ is the area of the peak associated to aliphatic $(2.42-2.79 \mathrm{ppm})$ and aromatic $(8.14-8.17 \mathrm{ppm})$ dicarboxylic units.

${ }^{\mathrm{b}}$ Degree of randomness evaluated as: $\left(A_{1.90-2.10-1.90} / 2\right) / A_{2.42-2.79+}\left(A_{1.90-2.10} / 2\right) / A_{8.14-8.17}$. 
Table 2. Non-isothermal crystallization parameters for pure copolyesters and their nanocomposites.

\begin{tabular}{ccccc}
\hline Sample & $\boldsymbol{\Phi} /{ }^{\mathbf{C}} \mathbf{~} \mathbf{~ m i n}^{-\mathbf{1}}$ & $\boldsymbol{T}_{\mathbf{0}} /{ }^{\mathbf{}} \mathbf{C}$ & $\boldsymbol{T}_{\mathbf{c}}{ }^{\mathbf{O}} \mathbf{C}$ & $\boldsymbol{\Delta} \boldsymbol{H} / \mathbf{J ~ g ~}^{-\mathbf{1}}$ \\
\hline 5 & 160 & 131 & 14.5 \\
10 & 156 & 126 & 15.4 \\
15 & 153 & 122 & 16.4 \\
20 & 150 & 120 & 16.8 \\
\hline 5 & 168 & 132 & 17.6 \\
10 & 159 & 127 & 17.3 \\
15 & 154 & 124 & 17.2 \\
20 & 151 & 121 & 16.9 \\
\hline 5 & 165 & 143 & 15.0 \\
10 & 163 & 138 & 16.1 \\
15 & 160 & 135 & 18.0 \\
20 & 157 & 133 & 18.3 \\
\hline 5 & 161 & 141 & 12.7 \\
10 & 160 & 136 & 16.8 \\
15 & 158 & 134 & 18.3 \\
20 & 157 & 132 & 18.2 \\
\hline 5 & 170 & 142 & 15.0 \\
10 & 168 & 137 & 16.6 \\
15 & 165 & 134 & 18.3 \\
20 & 160 & 132 & 20.0 \\
\hline 5 & 159 & 131 & 11.2 \\
10 & 157 & 126 & 13.6 \\
15 & 155 & 123 & 15.5 \\
20 & 152 & 120 & 15.5 \\
\hline & & &
\end{tabular}


Table 3. Non-isothermal kinetic parameters for all studied samples.

\begin{tabular}{|c|c|c|c|c|c|c|}
\hline Sample & $n$ & $\mathrm{Z}_{\mathrm{t}} / \mathbf{m i n}^{-n}$ & $k / \min ^{-1}$ & $Z_{\mathrm{c}}$ & $t_{1 / 2} / \mathrm{min}$ & $t_{1 / 2}^{-1} / \mathrm{min}^{-1}$ \\
\hline 5 & 3.2 & 0.004 & 0.18 & 0.33 & 5.00 & 0.20 \\
\hline 10 & 3.6 & 0.018 & 0.33 & 0.67 & 2.74 & 0.36 \\
\hline 15 & 3.8 & 0.041 & 0.44 & 0.81 & 2.09 & 0.48 \\
\hline 20 & 4.0 & 0.085 & 0.54 & 0.88 & 1.68 & 0.60 \\
\hline 5 & 3.1 & 0.003 & 0.15 & 0.3 & 5.84 & 0.17 \\
\hline 10 & 3.6 & 0.013 & 0.29 & 0.65 & 3.08 & 0.32 \\
\hline 15 & 3.9 & 0.060 & 0.49 & 0.83 & 1.87 & 0.53 \\
\hline 20 & 4.1 & 0.178 & 0.66 & 0.92 & 1.38 & 0.72 \\
\hline 5 & 3.2 & 0.004 & 0.18 & 0.34 & 4.93 & 0.20 \\
\hline 10 & 3.6 & 0.025 & 0.36 & 0.69 & 2.53 & 0.40 \\
\hline 15 & 3.8 & 0.053 & 0.46 & 0.82 & 1.96 & 0.51 \\
\hline 20 & 4.1 & 0.200 & 0.68 & 0.92 & 1.35 & 0.74 \\
\hline 5 & 3.2 & 0.008 & 0.22 & 0.38 & 4.00 & 0.25 \\
\hline 10 & 3.6 & 0.024 & 0.35 & 0.70 & 2.57 & 0.40 \\
\hline 15 & 3.8 & 0.050 & 0.46 & 0.82 & 1.99 & 0.50 \\
\hline 20 & 4.0 & 0.157 & 0.63 & 0.91 & 1.44 & 0.70 \\
\hline 5 & 3.2 & 0.003 & 0.15 & 0.30 & 5.89 & 0.17 \\
\hline 10 & 3.5 & 0.011 & 0.28 & 0.64 & 3.27 & 0.31 \\
\hline 15 & 4.0 & 0.086 & 054 & 0.85 & 1.69 & 0.60 \\
\hline 20 & 4.3 & 0.431 & 0.82 & 0.96 & 1.12 & 0.90 \\
\hline 5 & 3.2 & 0.009 & 0.24 & 0.4 & 3.80 & 0.26 \\
\hline 10 & 3.6 & 0.025 & 0.36 & 0.70 & 2.48 & 0.40 \\
\hline 15 & 3.8 & 0.039 & 0.43 & 0.81 & 2.12 & 0.47 \\
\hline 20 & 4.1 & 0.132 & 0.61 & 0.90 & 1.51 & 0.66 \\
\hline
\end{tabular}


Table 4. Non-isothermal crystallization kinetic parameters of copolyesters and their nanocomposites at different relative degrees of crystallinity as determined by the Mo equation.

\begin{tabular}{|c|c|c|c|c|}
\hline Sample & $\alpha_{t} t \%$ & $\ln F(T)$ & $a$ & $r^{2}$ \\
\hline & 20 & 3.42 & 1.33 & 0.9955 \\
\hline & 40 & 3.60 & 1.28 & 0.9961 \\
\hline & 50 & 3.66 & 1.27 & 0.9962 \\
\hline & 60 & 3.69 & 1.25 & 0.9962 \\
\hline & 80 & 3.78 & 1.23 & 0.9952 \\
\hline & 20 & 3.11 & 0.95 & 0.9905 \\
\hline & 40 & 3.28 & 0.96 & 0.9930 \\
\hline & 50 & 3.34 & 0.96 & 0.9940 \\
\hline & 60 & 3.39 & 0.97 & 0.9952 \\
\hline & 80 & 3.48 & 0.96 & 0.9970 \\
\hline & 20 & 3.13 & 1.15 & 0.9856 \\
\hline & 40 & 3.33 & 1.15 & 0.9903 \\
\hline & 50 & 3.37 & 1.13 & 0.9896 \\
\hline & 60 & 3.43 & 1.11 & 0.9918 \\
\hline & 80 & 3.51 & 1.04 & 0.9934 \\
\hline & 20 & 3.32 & 1.52 & 0.9754 \\
\hline & 40 & 3.53 & 1.44 & 0.9844 \\
\hline & 50 & 3.58 & 1.40 & 0.9836 \\
\hline & 60 & 3.64 & 1.37 & 0.9871 \\
\hline & 80 & 3.74 & 1.30 & 0.9901 \\
\hline & 20 & 2.96 & 0.82 & 0.9661 \\
\hline & 40 & 3.10 & 0.83 & 0.9721 \\
\hline & 50 & 3.15 & 0.84 & 0.9756 \\
\hline & 60 & 3.19 & 0.83 & 0.9794 \\
\hline & 80 & 3.26 & 0.80 & 0.9786 \\
\hline & 20 & 3.47 & 1.79 & 0.9226 \\
\hline & 40 & 3.69 & 1.61 & 0.9596 \\
\hline & 50 & 3.74 & 1.55 & 0.9662 \\
\hline & 60 & 3.80 & 1.51 & 0.9728 \\
\hline & 80 & 3.88 & 1.43 & 0.9836 \\
\hline
\end{tabular}


Table 5. Values of the Lauritzen-Hoffman parameters as deduced from the Nakamura method.

\begin{tabular}{cccc}
\hline Sample & $\left(\boldsymbol{t}_{\left.\mathbf{1} / \mathbf{2}^{-\mathbf{1}}\right)_{\mathbf{0}} / \mathbf{m i n}^{\mathbf{- 1}}}\right.$ & $\boldsymbol{K}_{\mathbf{g}} / \mathbf{K}^{\mathbf{2}}$ & $\boldsymbol{r}^{\mathbf{2}}$ \\
\hline PBST & $4.3 \mathrm{E}^{4}$ & $6.5 \mathrm{E}^{4}$ & 0.9707 \\
PBST-nHAp & $2.7 \mathrm{E}^{4}$ & $5.8 \mathrm{E}^{4}$ & 0.9826 \\
PBAdT & $0.06 \mathrm{E}^{4}$ & $2.8 \mathrm{E}^{4}$ & 0.9616 \\
PBAdT-nHAp & $0.3 \mathrm{E}^{4}$ & $4.1 \mathrm{E}^{4}$ & 0.9822 \\
PBSeT & $0.1 \mathrm{E}^{4}$ & $3.2 \mathrm{E}^{4}$ & 0.9872 \\
PBSeT-nHAp & $0.9 \mathrm{E}^{4}$ & $6.7 \mathrm{E}^{4}$ & 0.9882 \\
\hline
\end{tabular}


Table 6. Values of the secondary nucleation constants for regimens II and III, deduced from the Vyazovkin method.

\begin{tabular}{ccc}
\hline Sample & \multicolumn{1}{c}{$\boldsymbol{K}_{\mathbf{g}} / \mathbf{K}^{\mathbf{2} \mathbf{a}}$} & $\boldsymbol{r}^{\mathbf{2}}$ \\
\hline PBST & $6.8 \mathrm{E}^{4}, 1.4 \mathrm{E}^{5}$ & 0.9876 \\
PBST-nHAp & $6.5 \mathrm{E}^{4}, 1.3 \mathrm{E}^{5}$ & 0.9826 \\
PBAdT & $6.5 \mathrm{E}^{4}, 1.3 \mathrm{E}^{5}$ & 0.9713 \\
PBAdT-nHAp & $6.8 \mathrm{E}^{4}, 1.4 \mathrm{E}^{5}$ & 0.9889 \\
PBSeT & $4.5 \mathrm{E}^{4}, 1.0 \mathrm{E}^{5}$ & 0.9868 \\
PBSeT-nHAp & $7.2 \mathrm{E}^{4}, 1.5 \mathrm{E}^{5}$ & 0.9835 \\
\hline
\end{tabular}

${ }^{a}$ Secondary nucleation constants for regimens II and III appear in the left and right side, respectively. 
a)
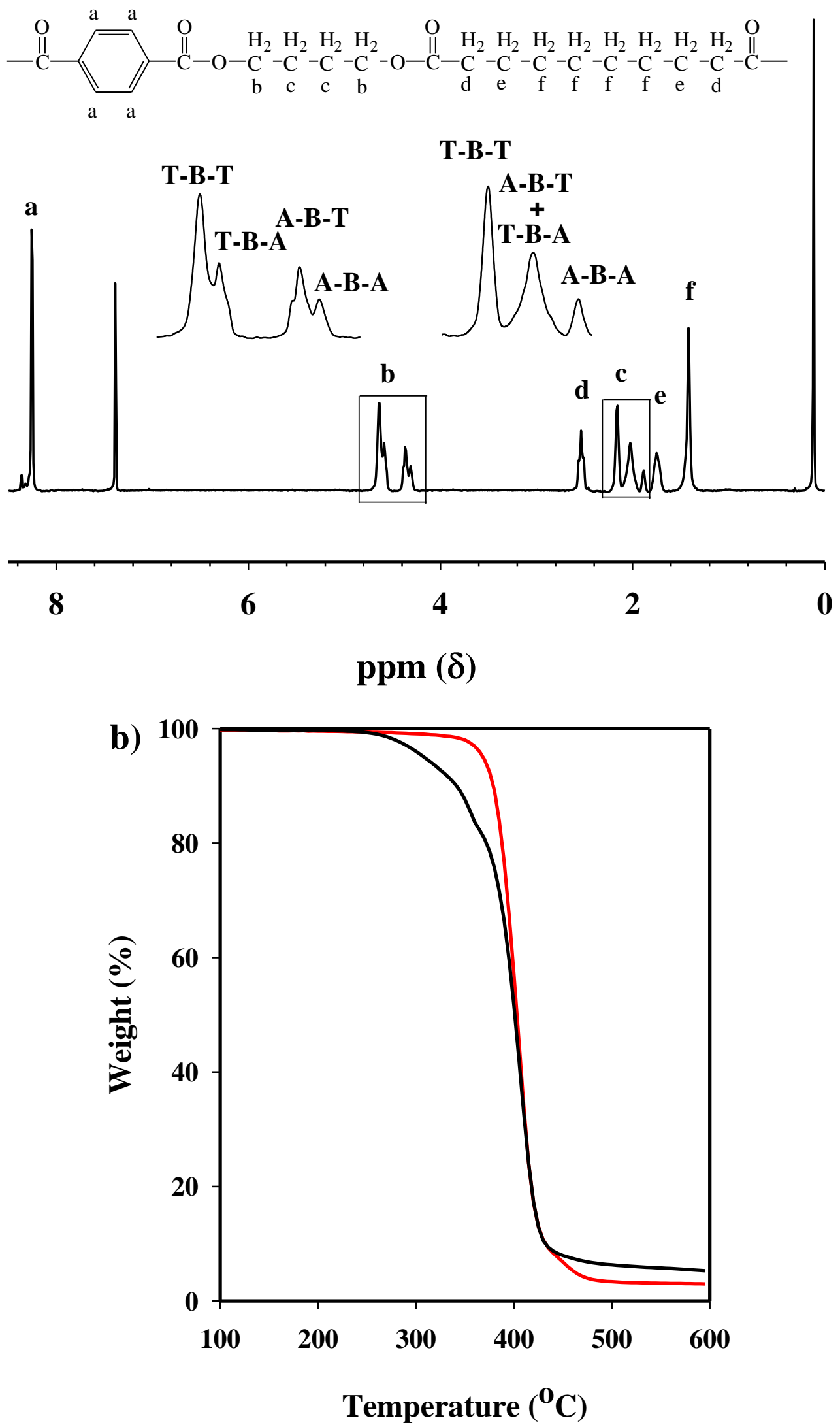

Figure 1

Heidarzadeh et al. 


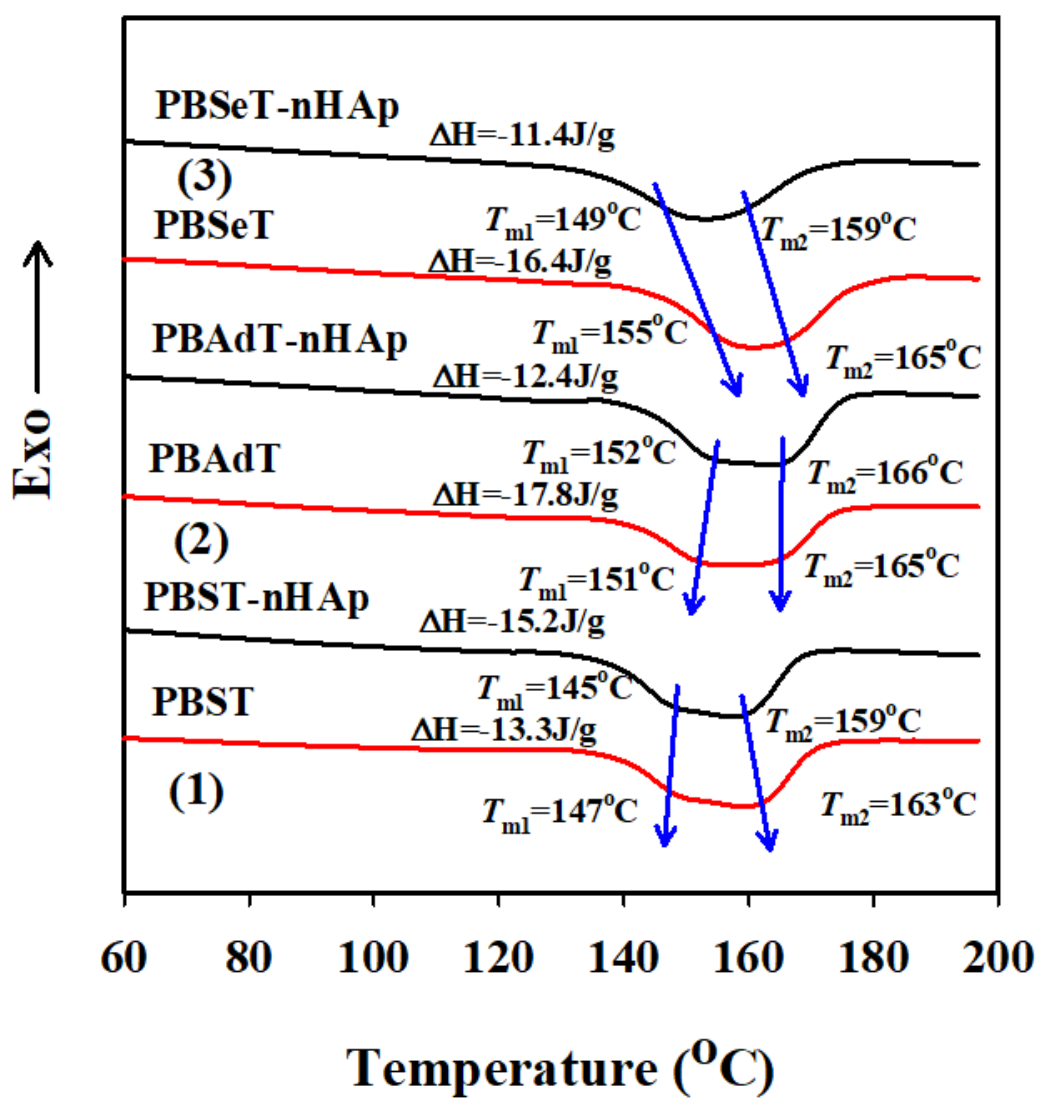

Figure 2

Heidarzadeh et al. 

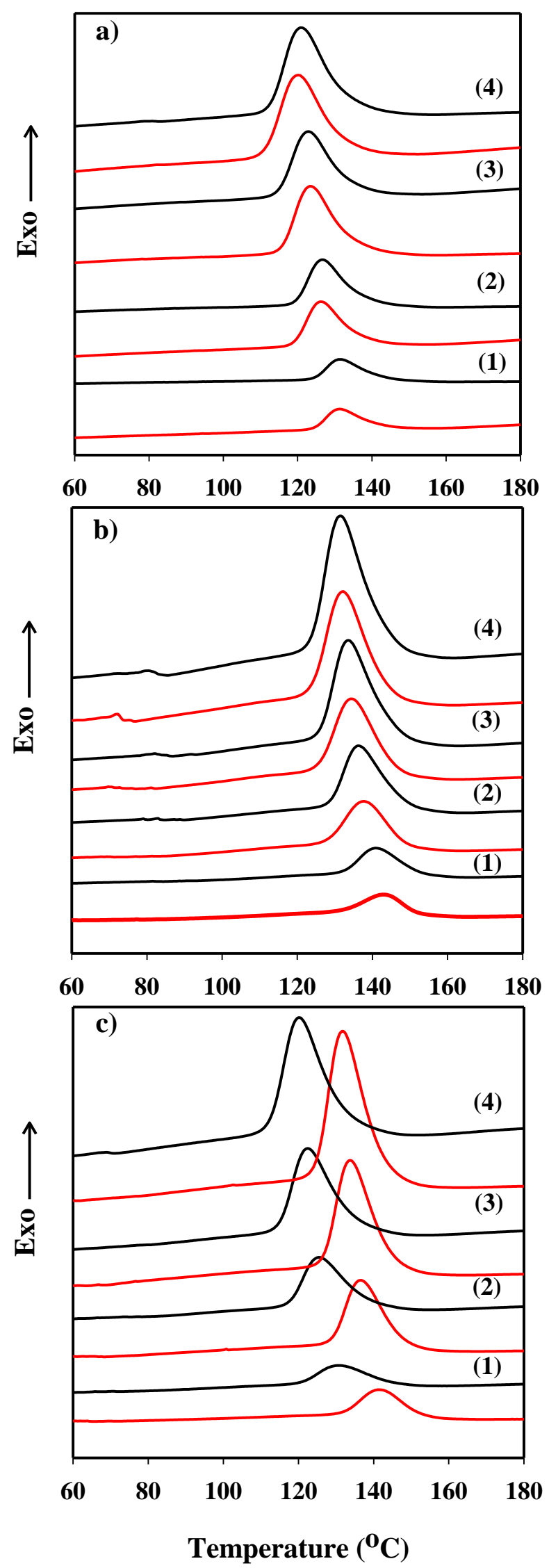

Figure 3

Heidarzadeh et al. 

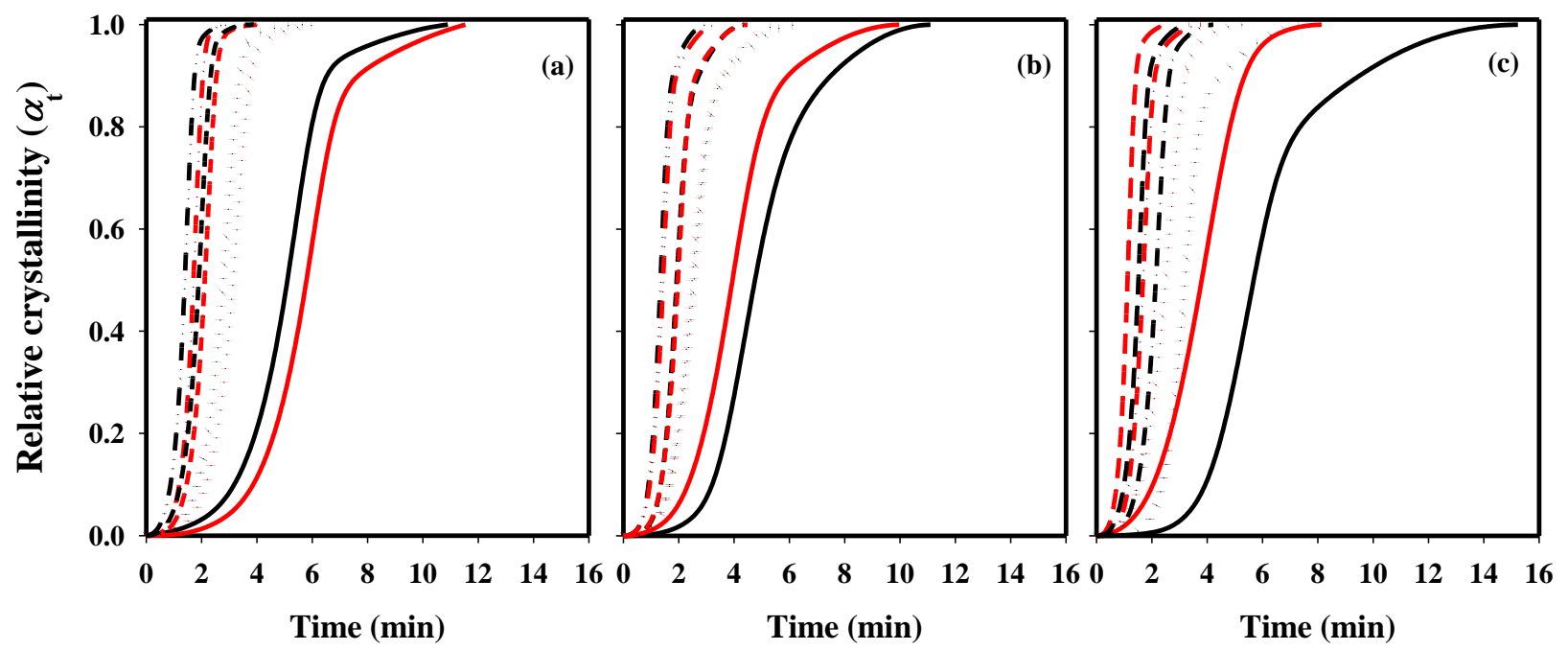

Figure 4

Heidarzadeh et al. 

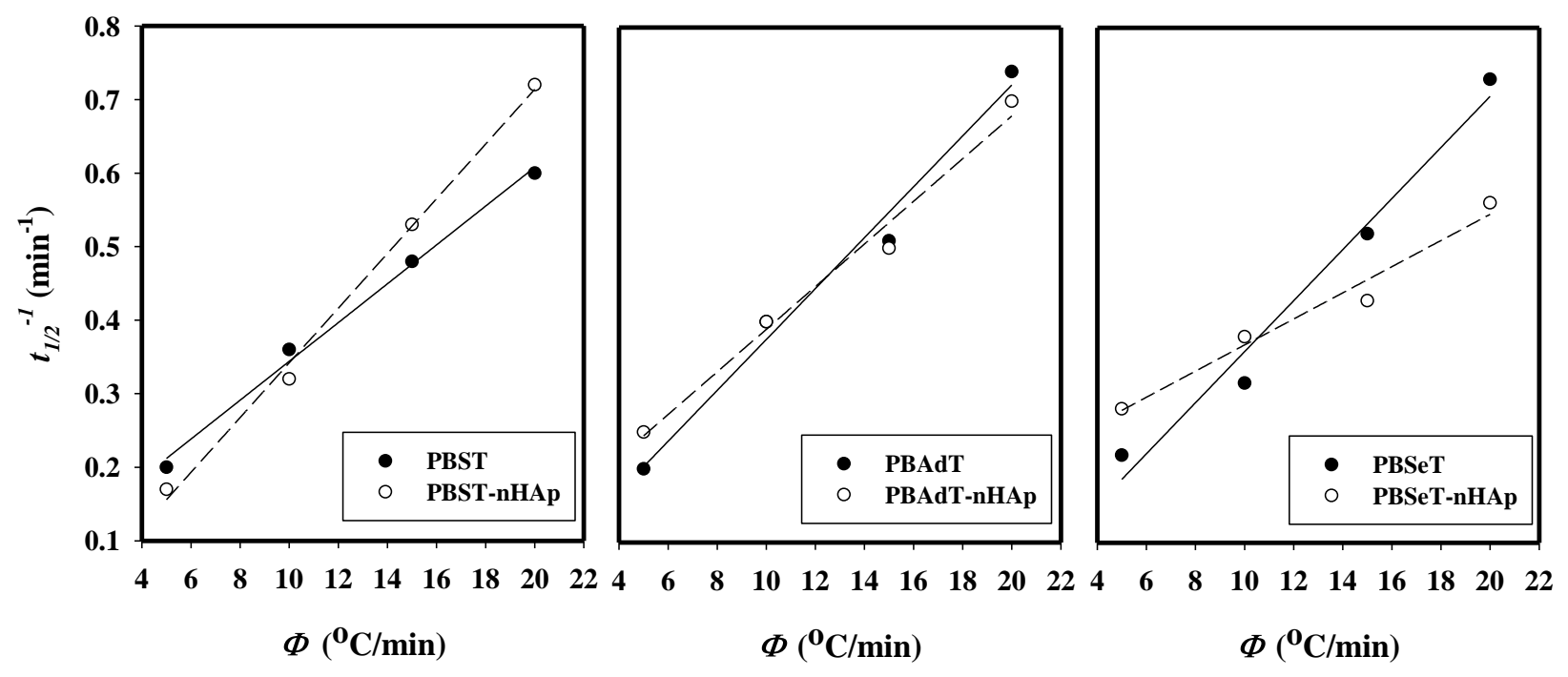

Figure 5

Heidarzadeh et al. 

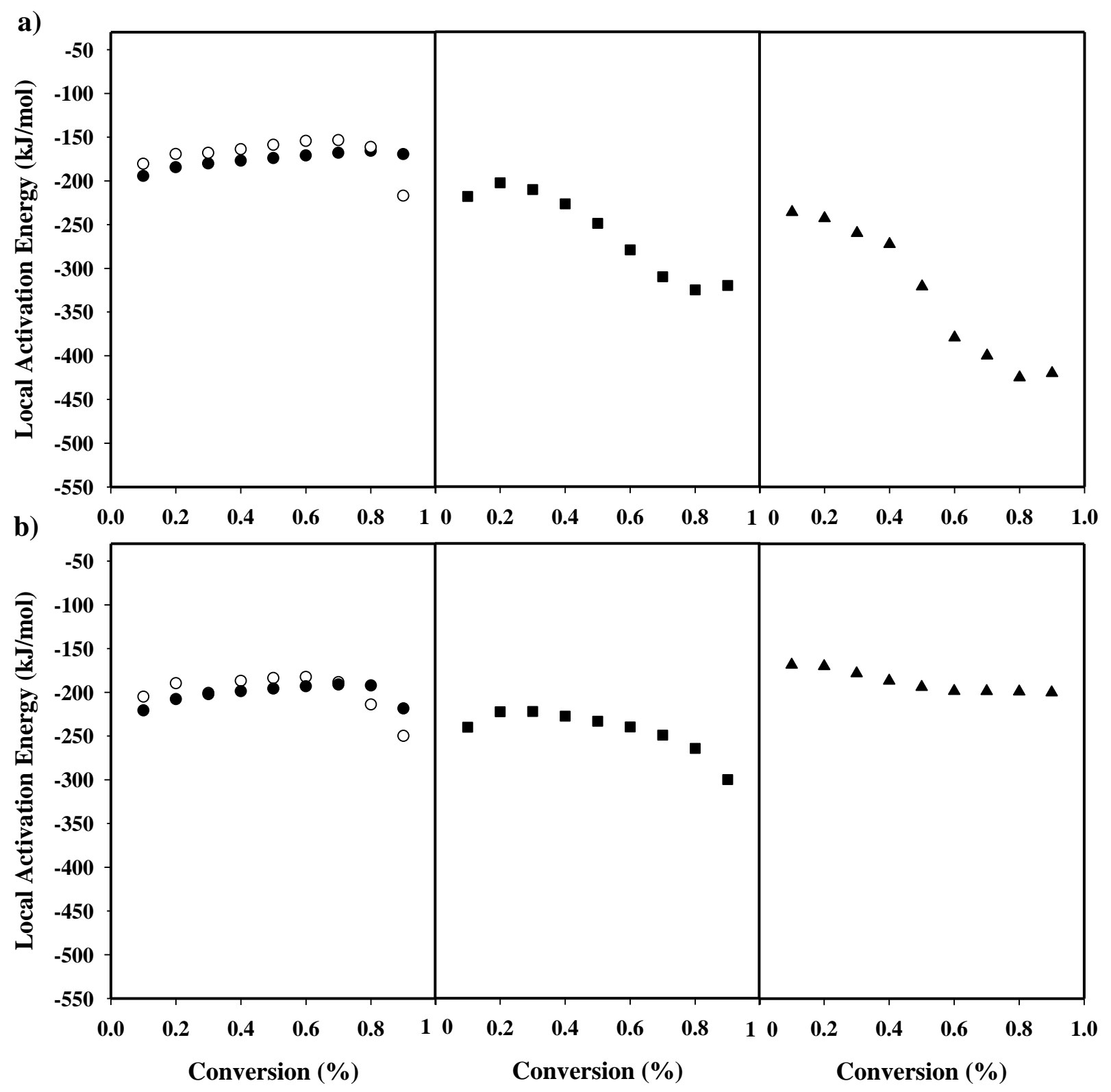

Figure 6

Heidarzadeh et al. 

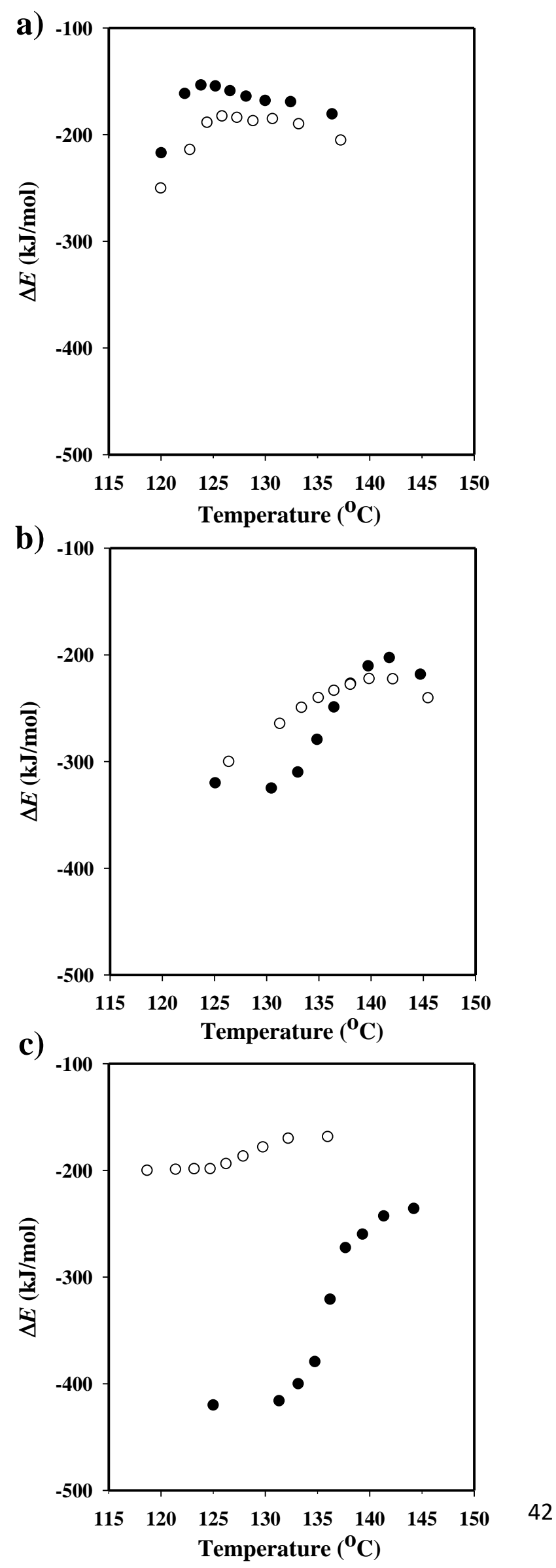

Figure 7

Heidarzadeh et al. 


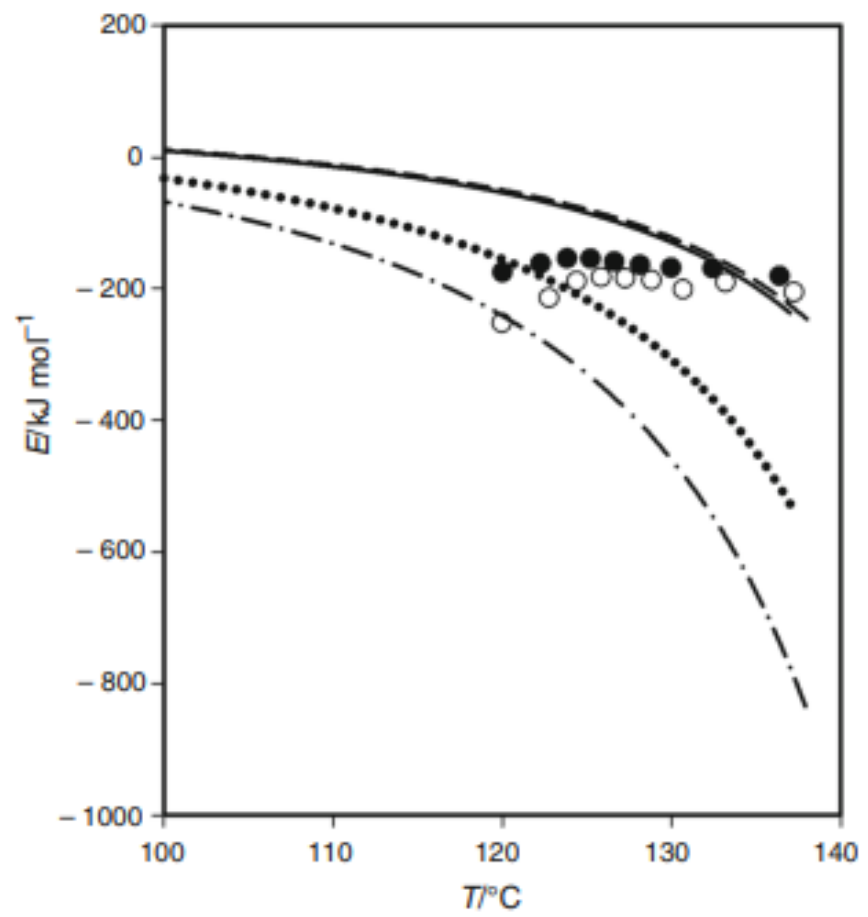

Figure 8

Heidarzadeh et al. 